\title{
A Study on Chinese Continuous Four-character Collocations and Their Translation into English Strategies
}

\author{
Weidong Yang (Corresponding author) \\ Foreign Languages Department, China University of Petroleum, 18 Fuxue Road, Beijing 102249, China \\ Email: clarkdream@sina.com
}

\author{
Weiping Dai \\ Foreign Languages Department, China University of Petroleum, Beijing, 18 Fuxue Road, Beijing 102249, China \\ Email: dwping@cup.edu.cn \\ Lijia Gao \\ Foreign Languages School, Jilin University, 2699 Qianjin Road, Chaoyang District, Changchun 300012, China \\ Email: tianping@jlu.edu.cn
}

\begin{abstract}
The Chinese continuous four-character collocations (CCFCCs) are vivid, concise and aesthetic. They have rich meanings, gorgeous literal talent, consistency and strong rhetorical effect. The CCFCCs are embedded in different sentences. They make the discourse more concise, lively, humorous and thought-provoking. There are many papers concerning the techniques and skills of translating four-character collocations, therefore, the present paper mainly focus on the translation strategies of CCFCCs.
\end{abstract}

Index Terms - Chinese continuous four-character collocations, idioms, translation

\section{INTRODUCTION}

Idioms are the essence of language. Languages see their best manifestations of colorfulness, forcefulness, terseness and richness in idioms. Therefore, the familiarization and grasp of idiom is widely regarded as the basic standard by which the language capacity is tested. However, what confronts the language learners is not only the great quantity of the idioms but also the tremendous hardness of how to use the idioms properly. Many experts and scholars have carried on their research in this field, especially in the field of Chinese idioms. The traditional views of idioms treat idioms as arbitrary and fixed pattern of languages. Many linguists regard idioms as non-compositional by definitions. They have focused on the techniques of translating Chinese idioms into English and the problems of translating them, like the accuracy of target language, the national characteristics and the manner of speaking and so on.

When they find it is not enough to analyze the Chinese idioms from the techniques, they began to discuss this question from different perspectives. From the macro point of view, there are people using the cognitive linguistics to study the acquisition of Chinese idioms (Zuo, 2006), applying socio-semiotic approach to the translation of Chinese four-character idioms (Zhang, 2006), etc. From the micro point of view, the most significance of Chinese idiom-translation studies is the translation of "A Dream of Red Mansions". Chinese idiom translation is mostly done in the translation of literal works. Other aspects are from the grammar perspective like subject-predicate structure and parallelism, analyzing the numeral of Chinese four-character idioms (Lin, 2008) and "The Symbolic Meanings of the 12 Symbolic Animals in Chinese Idioms" (Ma, 2006) and so on. Whatever the main theme is, these papers all analyzed the four-character collocations from ways like free translation, literal translation, using techniques to translation and so on.

Strictly speaking, Chinese four-character collocations cannot completely be the same as idioms, since the latter still has five-character collocations or even six or seven characters. However, most of the idioms we use today are the four-character ones. So, the present paper focuses on the four-character collocations from the idioms' perspective.

Chinese people like to use four-character collocations. According to Mr. Lv Shuxiang, the four-syllable collocations perhaps are the favorite forms by Chinese users (Wang, 2007). There are certainly some reasons for it. First of all, it is determined by the characteristics of Chinese. The Chinese syntax is usually flexible, which means it can make up many phrases with fixed number of words, such as four-character collocations. Secondly, the four-character collocations have the characteristics of balanced structure, and concise wording. And Chinese people would overstress the aesthetics of balance, as a result, they often favor four-character collocations,.

\section{SOURCES OF CHINESE FouR-CHARACTER COLLOCATIONS}


Four-character collocation is a broad concept. It can be classified into two categories: narrow and broad.

In the narrow sense, four-character collocations are quite the same as idioms. They are fixed and cannot be reformed. Every four-character idiom has its own story, if you cannot understand the story, then you cannot understand what they mean.

In the broad sense, any phrase or collocation which is made up by four characters can be covered in this category. Most of these collocations are the brand new ones made not long ago. They are unfixed ones and can be made into whatever types as long as they have four characters. Along with the development of our society, the four-character collocations also develop themselves, examples could be cited and translated in English as "one country two systems", "people first", "harmonious society", "environmentally-friendly products", "Hope Project" and so on.

Many Chinese idioms are passed down from ancient times and have been in existence for quite a long time. Chinese idioms mainly originate from the oral folk idioms, and their minor source is the creations of literators of different times, or from the written materials. However, like other languages, Chinese has also absorbed many loan words, including some idioms. So another noticeable source may be the loan words, or idioms from other languages.

\section{A. Daily Experiences of Ordinary People}

Most of the idioms and idiomatic expressions in Chinese are from the common people, that is, from their occupations and hobbies. As in the case of words, idioms should be counted on the man-of-letters group for their creation. The best of our idioms, like those of words, come from factories, kitchens and farms rather than from libraries, living rooms or theaters. From ancient times till now, folk idioms are forever the main stream of idioms as they are lively and vivid expressions of people's daily life. These idioms with simple words put together, however, contain profound meanings and reflect life experience. In the civilization process of human beings, different geographical conditions, customs, ways of living or even some historical events have had great influence upon the evolution of language and have explicit signs on it. For example, China is a traditional agricultural country. A great number of idioms and idiomatic expressions relating to agriculture and farm work have been widely accepted by its people.

For examples (English versions):

when water flows, a channel is formed

put aside the armor and pick up the hoe

one crop was eaten when the next is still green

\section{B. Written Materials}

Many oral folk idioms, after selection and polishing, have been written down by literators, and become the written idioms passed down to this day. Those idioms from written materials again have different origins, which in general can fall into the following four categories:

i. Mythology

Chinese ancestors have created many beautiful mythologies, some of which reflect their strong will to conquer the nature, e.g. "the man who does not give up can move mountains" (English version).

ii. Fable

Fables and mythologies are quite alike, but the differences lie in that the former are mostly created by literators and endowed with didactical purpose while the latter are the collective works of common people who naturally express a certain kind of thoughts without definite didactical purpose, e.g. "Kua Fu's race with the sun" (English translation).

iii. Historical event

Some idioms have their historical backgrounds or contain allusion in them, e.g. "be besieged on all sides" (English translation); "when the map was unrolled, the dagger was revealed" (English translation)— the real intention is revealed in the end.

iv. Creation of literators

Literary language initially came from the folk language, some of which have undergone the polishing of literati and spreading of literary works and have finally entered daily languages and become idioms, e.g. "a friendship formed in childhood" (English translation)—__ said of boys and girls.

\section{Foreign Idioms Borrowed into Chinese}

Since the Opium War (1840-1842), quite a number of new words have been introduced into the Chinese language from western countries. Foreign idioms, to be specific, also originated from the sources as mentioned above, i.e. mythologies, fables, historical events and creations of literators. However, the foreign idioms only take up to no more than $1 \%$ of the total number of Chinese idioms (Shi, 1979). Mr. Shi explains in his A Study on Chinese Four-Character Collocations that since the Chinese language is always rich in idioms, we can't help thinking of a Chinese idiom which is equivalent in meaning to a foreign idiom in the process of translation and our native idiom is habitually adopted to save the trouble of translation, resulting in the relatively small number of the foreign idioms assimilated and accepted in the Chinese language. Therefore, we tend to use the existing Chinese idioms to translate some foreign idioms. For examples, to help a lame dog over a stile (English translation), like a rat in a hole (English translation), to cast pearls before swine (English translation), a black sheep (English translation), to paint the lily (English translation), etc. 


\section{FeATURES OF CHINESE FOUR-CHARACTER COLLOCATIONS}

As special language forms, idioms are derived from those words or phrases that have been used as a whole by the people for a long period of time. They can be explained with words of the contemporary language and they are frequently used in the daily life. However, their constructions and vocabulary may not be the same as that of the contemporary language. As fixed word structures with special and integral meanings established despite of long-term usage and common practice, idioms inevitably have their distinctive features.

\section{A. National Character}

Idioms are created by the folk in labor and they have a close relationship with human beings and their environments. To be specific, idioms are closely related to the geographic and historical backgrounds, business activities, customs, religious beliefs and cultural psychology of its nation. Therefore, a large number of Chinese idioms contain the flavor of national culture; hence they can be called nation-specific or culture-specific idioms.

For example, the ancient Chinese people regarded the heart as the center of the soul, thinking and feelings of human beings and thus they created a great number of idioms related to "heart", such as, absent-minded, have a deep longing for, be perfectly content, disease in one's vital organ_-danger from within, one's heart is like dead ashes_hopelessly apathetic.

Another prominent feature is the allusive idioms. We can find many allusions contained in some idioms, from mythologies, fables, historical events, etc., such as "Dongshi, an ugly woman, knitting her brows in imitation of the famous beauty Xishi, only to make herself uglier" (English translation) crude imitation with ludicrous effect, "proffer a birch and ask for a flogging" (English translation)__ offer a humble apology, "learn the Handan walk" (English translation)__ in trying to acquire a new trick, lose the ability one already has, "whirl the hatchet with a noise like the wind" (English translation)__ an uncanny feat.

Allusive idioms are mostly rich in cultural deposits or national flavor, which may puzzle foreigners in the intercultural communication if they are not familiar with the stories behind these Chinese idioms.

For example, without understanding the story about Qin Hui and his wife, Wang Shi's conspiracy under the eastern window to murder Yue Fei, it would be hard to understand that "the cat is out of the bag" (English translation) means "to be exposed".

\section{B. Semantic Integrity}

A Chinese idiom functions as a single semantic unit. In other words, it is used as a substantive part of the language. Although composed of several characters, the meaning of a Chinese idiom is not the sum added by the meanings of each individual character. The idiom must be used as an integral unit in the conversation or writing. Generally speaking, almost every Chinese idiom has two meanings, say, denotative meaning, and connotative meaning. The soul of Chinese idioms lies in their connotation, without which they will be disqualified as idioms. The meaning of a Chinese idiom is more than the sum of the meanings of its individual elements.

For example, the literal meaning of "wind, flower, snow and moon" in Chinese is actually "referring originally to certain types of literary works and later to effete and sentimental writings in general" in English; the literal meaning of "having a bamboo in one's chest" in Chinese refers to "having a well-through-out plan" in English.

\section{Structural Features}

About $97 \%$ of Chinese idioms are constructed with four characters (Feng, 2002), so they are often called four-character idioms. Unlike any other language form, four-character idiom is the unique feature of the Chinese language. China seems to be a nation whose people traditionally favor even numbers to odd numbers and lay great emphasis on symmetry, parallelism and antithesis, which is an important cultural mentality unparalleled elsewhere. An idiom of four characters can be quite elegant, while a three-character idiom is a bit vulgar. Therefore, Chinese four-character idioms are fossilized patterns of language, which allow little variation in form. They generally resist random replacement. In the Chinese language, phrases, according to their different grammatical structures, are classified into doublets, SV (subject + verb) phrase, VO (verb + object) phrase, MrMd (modifier-modified) phrase, etc. Since idioms are fixed phrases, they can also be classified into doublets, SV structure, VO structure, MrMd structure, etc.

\section{Rhetorical Features}

Rhetoric is, perhaps, the most distinctive feature of Chinese four-character idioms. Abundant with various rhetorical devices, such as metaphor, parallelism and rhyme, many four-character idioms are easy to memorize and have been handed down from ancient times and into present existence of today. As a special form of language, Chinese four-character idioms have the aesthetic function which conveys the aesthetic information by means of its various rhetorical devices.

a. Simile

the courtyard is as crowded as a marketplace (English translation)

as firm as a rock (English translation)

b. Metaphor

an incessant stream of horses and carriages (English translation)— _ heavy traffic 
every bush and tree looking like an enemy soldier (English translation)_ _ a state of extreme suspicion and fear c. Metonymy

dark willows and blooming flowers (English translation)__ _ a beautiful scene

jackals and wolves hold sway/the cruel and wicked are in power (English translation)

d. Hyperbole

with three heads and six arms (English translation)—_ superhuman powers

each word worth a thousand pieces of gold (English translation)—_a highly finished literary product

e. Euphony

Chinese four-character idioms are usually masterpieces of the art of euphony in that many of them not only have the symmetrical semantic structure but also adopt rhyme or alliteration, which makes them a feast for both the eyes and ears.

About $40.8 \%$ of all the Chinese four-character idioms are semantically symmetrical or partially symmetrical, which shows that parallelism is a distinctive rhetorical device favored in Chinese idioms.

\section{Chinese Continuous Four-Character Collocations (CCFCC) AND Their Translations}

The Chinese four-character collocations can express a kind of meaning concisely with highly symmetrical structure. The language is simple with profound meaning. Though they are concise, they contain meaningful expressions. With them, we can explain the profound truth. They are expressive with live images. If we use them appropriately, they can enhance the language expressiveness.

Like those four-character collocations, the continuous four-character collocations are also vivid, concise and aesthetic. What's more, they have rich meanings, gorgeous literal talent, consistency and strong rhetorical effect. These CCFCCs are embedded in different sentences and make the sentences wonderful. They make our discourse more concise, lively, humorous and thought-provoking.

\section{A. Differences in Terms of Translating CCFCC}

Chinese traditional culture is known as equilibrium and well-balanced proportion. In addition, this traditional aesthetic standard also exerts an imperceptible influence on Chinese language and embodies the idea in the language form of Chinese four-character structure. The Chinese four-character structure contains stable syllables and unified wordage, with the strong characteristics of neat structure and harmonious sounds. The four-character structures can be seen in almost all literal forms. They can be used as single sentences as well. They are so esthetic that they mix the syllable and form features of Chinese characters ingeniously. When we are reading them, we feel they are harmonious and beautiful, concise and comprehensive. Nevertheless, the Chinese continuous four-character structure has extremely strong effect in comprehensive meanings, magnificent literal talent and coherent tone.

Although the literal meaning of the Chinese continuous four-character structure is not distinctively enough in this way, it stresses imposing manner and frame of mind, often having ideological and practical meaning. While on the other hand, English is a rather rational language. It pays attention to clearness and avoids the disorder of logic and padding of words. Consequently, it is unavoidable that the careful and neat parallelism structure in Chinese turns out to be uneven in English. Therefore, we cannot insist on keeping the form identical with the source language. Otherwise the target language will become slack with words that just string together, which is not appropriate in the standard of English expression. For this reason, when we translate the collocations of Chinese four-character structure into English, we can transfer them into appropriate structure in English, according to their semantic and logic elements.

There are so many papers talking about the techniques to translate four-character collocations, such as in the field of literal translation, free translation or using equivalents to translate, and so on, the present paper here will mainly concentrate on the translation of CCFCC.

\section{Coordinate relations}

The continuous four-character structure of parallel relation usually can be transferred into the following structures:

a. adjective + noun

The valley of the Grand Bend is full of multi-peaked mountains, joined canyons and turbulent waters. (English translation)

b. noun/adjective + in + noun

These silk blouses are made of pure silk of best quality. They are moderate in price, excellent in craftsmanship and unique in designs. (English translation)

c. Prepositional phrases

The Yangzi River is very much awe-inspiring with deep riverbeds, rushing currents and steep banks. (English translation)

d. Noun phrases

We should vigorously foster the fine esprit, including dedication to work, and offering convenience and fine services to the masses. (English translation)

e. Verb phrases

We should emancipate our minds, seek truth from facts and work hard creatively. (English translation) 
2. Subject and subordinate relation

There are many CCFCCs that describe around one central idea. Therefore, when translating them into English, we can transfer them into adverbial or adjunct structure, for example:

The factory can produce various new types of buttons in thousands of different designs for coats, suits, fashions, shirts and sweaters. (English translation)

In addition, some CCFCCs can be treated as single sentences. When translating them, we can transfer the main part into the subject and the subordinate into the other parts of the sentence, or even change the whole structure of the sentence, for example:

So many men and women with high ideals, worried and frustrated, wish to do something about it, only to find that, able as they were, they had no chance to carry out their ideals. (English translation)

3. Casual relations

Some CCFCCs contained recessive casual relations; therefore, we put the part which indicates the reasons ahead when transferring them. Then, we use verbs like "form" and "achieve" to connect with the rest parts of the sentence, for example:

There are many hidden shoals in the river, presenting a challenge to navigators. And the surges on the swift current and roars of the terrifying waves achieve great momentum. (English translation)

\section{B. Strategies in Translating CCFCC}

\section{Literal and free translation}

People often talk about literal translation and free translation, discussing which way of translation is better to use to translate a sentence. The target language must express what source language means, distortion is not allowed. Literal translation and free translation are two main forms of translation. They are not repulsive, contrarily, they are complementary. Literal translation retains original skill. When literal translation is not okay, people would use free translation instead. Free translation expresses general idea of the original, and can be accepted by readers. A good translation composition contains both literal translation and free translation.

Literal translation and free translation are two basic skills of translation. Literal translation refers to translating a sentence originally, keeping the original message form, including construction of sentence, meaning of the original words, and metaphor of the original and so on. Translation would be fluent and easy to be comprehended by target language readers.

For examples (English translations): Peaches and plums do not have to talk, yet the world beats a path to them; Mountains can be removed and Oceans can be land filled, but one's nature can hardly be changed; and Past experience, if not forgotten, is a guide for future.

Free translation needs not pay attention to the form of the original, including construction of the original sentences, meaning of the original works, and metaphor of the original and so on. But free translation does not mean to delete or add content to the original and translators must treat the original carefully, know its connotation, translate it naturally, and express the meaning of the original. Free translation is a skill and translators must know the cultures of both source language and target language, and must have extensive knowledge.

For example: "No great loss without some small pain." or "A loss might turn out a gain." (English translation)

Literal translation and free translation play an important role in translation, though in specific circumstances, using equivalents to translate is also essential. Since in this way, the source language will be more like the native rather than the translated. Therefore, we cannot ignore its role. In the translating process, using equivalents to translate refers to the expression of adopting certain words, phrases or idioms of the source language into the target language. This way of translating is the most important and the commonly used method in translation. The following are the more examples (translated into English): as light as a feather, as steady as the mountain; it never rains but it pours; man proposes, God disposes.

We can use concrete and specific words or phrases to translate the abstract ones in the source language, in order to eliminate or reduce the linguistic differences in the translation process.

For example: 看菜吃饭, 量体裁衣, if we just translate it in the literal way, it will turn to be "to eat your rice according to the dishes served, measure the man before cutting the cloth", which certainly is wordy. Instead, we should translate it into "to fit the appetite to the dishes and the dress to the figure".

\section{Words' structure}

In terms of collocations, CCFCCs have various configurations. Analyzing the structure of CCFCC is an important means of thorough understanding of the connotation of CCFCCs. In accordance with the grammatical structure, CCFCCs can fall into the following types.

a. Subject-predicate structure

The Chinese subject-predicate structure is quite different from that of English. In the Chinese subject-predicate structure, the number of the subject does not change and there is no change in the tense or the number in the predicate. However, the English subject-predicate structure is the other way round. In general, when we translate the Chinese subject-predicate structure into English, we should also put it in a subject-predicate structure, but there are many exceptions, depending on the circumstances to require different treatments, such as subject-predicate structure. When 
we do the translation, we need to make some changes in order to highlight the four-character structures.

In many cases, the Chinese subject-predicate structure has the equivalent in English, such as 设备完善, 技术一流 and 色调雅致,图案新颖. The first one can be translated as “The facilities are perfect and the techniques are top-class". The latter can be translated as "The colors are elegant and the designs are novel."

The subject-predicate structure can become the predicate of the sentence in Chinese, while it is not appropriate in English. Therefore, it needs to be changed to "be + adjective + prepositional phrase", for example:

This carpet is elegant in color and novel in design. (English translation)

In order to highlight the magnificence of the subject-predicate structures, sometimes we just translate them into prepositional phrases directly, placing them in the first part of the sentences, for example:

With fine workmanship and novel design, the products manufactured in this factory reached the advanced level of the domestically-made bits of the kind. (English translation)

b. Verb-object structure

There can be various translations of Chinese verb-object structure according to its different position in the sentence. If the nouns and verbs in this structure are the predicate and object respectively in the source language, we can still translate them into verb-object structure in the target language. If this verb-object structure is the subject or object in the sentence, we often translate it into a noun phrase.

The Chinese verb-object structure can still remain in English, for example:

We should create more opportunities, provide better training, assist the laid-off employees in revising their job-seeking and encourage them to find new jobs on their own. (English translation)

If the verb-object structure is the subject or object in the sentence, it needs to be translated into a noun phrase. For example:

At the current stage, revitalization of the economy is our major task. (English translation)

c. Structure of modification

There are many modification structures in Chinese. The key words of the structure of modification are nouns or gerunds, which are usually the last part of the phrase. The words or phrases in front of the key words play the role of description, modification and limitation. For Chinese, we mainly focus on the collocation between the attribute, adverbial modifier, complement and the key word, whether it has appropriately expressed the relation between "modify and modified", "limit and limited", "restrict and restricted", "complement and complemented" and whether it can make the concept more clearly. While analyzing English structure, the main focus is on whether the preposition is used correctly and whether it is identical in the number, gender and case between modifiers and key words.

Generally speaking, the Chinese structure of modification can be translated into English with the following methods:

We adopt the structure of "adjective + noun" when translating the phrases of "modify and modified", for examples:

religious undertaking (English translation)

self-sufficient economy (English translation)

For the relation like "limit and limited", we often translate the second verb phrase into an adverbial purpose, for examples:

to draw cakes to relieve one's hunger (English translation)

to cut the feet to fit the shoes (English translation)

For the relation of "complement and complemented", the words or phrases that complement the key words should be translated into a prepositional phrase, for example:

to shift the blame onto others (English translation)

d. Coordinate structure

The translation of coordinate structure has the following situations:

In Chinese, we often use two familiar phrases together, such as 自私自利, 骄奢淫逸, in translating it into English, we may just translate one of these two, then the English version of the above phrase will be "self-centered and luxury loving”.

There are also some four-character collocations that only can express their meaning when the meaning of the four characters is translated as a whole. In this situation, we cannot only translate one or two words of the phrase; otherwise, we cannot understand what it means. For examples:

coincidentally (English translation)

travel extensively (English translation)

heretical sect (English translation)

3. Style

a. Literary works

The earliest CCFCCs probably emerged in the literal works and most CCFCCs that we are familiar with are from literature. The "four-character poetry" had ruled the entire world of poems as early as in the Zhou Dynasty. These poems have dominated the world for more than 1000 years. They contributed a lot to Chinese for making rich vocabularies. Most of them are so common that we almost hardly notice these four-character collocations were originally poems. In "The Book of Songs", almost all forms of four-character collocation had appeared. Besides, the novels in the Ming and Qing Dynasties possessed the largest number of four-character collocations, such as in The 
Journey to the West and A Dream of Red Mansions. CCFCCs, which belong to the literal work, usually have rich connotations. If we just translate them literally, the readers might not understand them. In this case, free translation with annotations will probably be better.

b. Practical writing of advertisements

Like other practical writings, the practical advertisement also demands a simple beauty of conciseness, a focus, and clear structure. Nevertheless, it has its own characteristics. It has the artistry, besides the succinctness. Advertisements can make people feel joyful and eager to buy the item for its artistic impression.

For example:

The business scope of Beijing Gongmei Group: metal arts and crafts, scientific and educational instruments, real estate, commerce and trade, environmental art, tourism services, advertisement and publication, culture and education. (English translation)

c. Practical writing of tourism

The practical writing of tourism includes various types, such as the advertisements of tourism, schedule, relative contract, introduction of tourist attractions and notices and so on. Take Introduction of Tourist Attractions as an instance.

The Introduction of Tourist Attractions can be in the form of both prose and explanation. Although they have their own characteristics, their main purpose is all the same, that is, to enhance attractiveness that can bring joyfulness to the readers and make them eager to see the spots as soon as possible. For example:

Since the $18^{\text {th }}$ century, after more than 200 years of excavation, it has been proved that Pompeii was a city of about twenty thousand people, most of whom were Roman nobles. Therefore, the houses were beautifully constructed, with all necessary facilities and in good order. (English translation)

\section{Difficulties in Translating CCFCC}

More than any other feature of language, the continuous four-character collocations demand that the translation be not only accurate but highly sensitive to the rhetorical nuances of the language. Therefore, the translation of CCFCC is an exacting act.

Once CCFCC has been understood and interpreted correctly, the next step is to decide how to translate it into the target language. The difficulties involved in translating CCFCC are totally different from those involved in interpreting them. Here the question is not whether the given CCFCC is transparent, opaque or misleading. An opaque expression may be easier to translate than a transparent one. The main difficulties involved in translation CCFCC may be summarized as follows:

a. CCFCC may have no equivalent in the target language.

The way a language chooses to express, or not express various meanings cannot be predicted and only occasionally matches the way another language chooses to express the same meanings. One language may express a given meaning by means of a single word, another may express it by means of a transparent fixed expression, and a third may express it by means of a four-character collocation, and so on. It is therefore unrealistic to expect to find equivalent words or phrases in the target language. It is not the specific items the expression contains but rather the meaning it conveys and its association with culture-specific contexts which can make it untranslatable or difficult to translate.

b. CCFCC may have the similar counterpart in the target language, but its context of use may be different.

For example:

The Chinese “一箭双雕” and English "kill two birds with one stone” have the similar meaning and image, however, English idiom "kill two birds with one stone" is always translated in 一举两得 in practice. Chinese idiom 一箭双雕 lays more emphasis on the aim. It contains some special feeling. It can express somewhat the style of ancient Chinese as well. Its usage is not as wide as 一举两得. In addition, 一石两鸟(the literal translation of “kill two birds with one stone") is already considered as an foreign idiom borrowed into Chinese idioms, and it is seldom used by the Chinese people.

"Kill two birds with one stone" can express the meaning of doing one thing, getting two good results, but the Chinese readers cannot get the connotation of “一箭双雕”, for it focuses on the purpose of the person. In short, although source language and target language may have similar idioms, sometimes they cannot have equal function.

\section{CONCLUSION AND IMPLICATIONS}

The Chinese four-character collocations are always regarded as the essence of language, and their familiarization and grasp of is widely regarded as the basic standard by which the language capacity is tested. However, due to their great quantity and tremendous hardness of how to use the expressions properly, the acquisition has always been a big problem confronting the language learners. Translation is very probably the most complex type of event yet produced in the evolution of the cosmos (Richards, 1953). It is also true of CCFCC translation. The challenging aspects of Chinese continuous four-character collocation translation lie in the distinct features of their constitution, namely, symmetrical form and rhetorical aesthetic function with culture-loaded elements such as allusion and metaphor.

The Chinese continuous four-character collocations are usually divided into the phrases category, nevertheless, this paper believes since they best manifest the expressiveness of the language, they contain the similar syntax as sentence or discourse do. Therefore, they can be analyzed in the same way, though there exist certain difficulties. 
Translating something does not just mean to translate the language. Instead, it means to translate the culture into another language. This paper is a tentative study of the features and translation method of CCFCC. The research should not be limited in the technique aspect, but should develop onto the levels of words' structure, collocation, grammar and style.

\section{REFERENCES}

[1] Bell, R.T. (1991). Translation and Translating: Theory and Practice. London: Longman.

[2] Fawcett, R. P. (1997). Translation and Language. Manchester: St. Jerome.

[3] Feng, Q. H. (2002). A Course in Practical Translation. Shanghai: Shanghai Foreign Language Education Press.

[4] Lin, Y. (2008). A Tentative Study on the Translation of Chinese Four-Character Collocations into English. Qufu Normal University.

[5] Ma, D. (2006). On the Symbolic Meanings of Twelve Symbolic Animals. Sichuan University.

[6] Newmark, P. (1989). The Translator's Handbook. London: Aslib.

[7] Richards, L.A. (1953). Toward a theory of translating: studies in Chinese thought. Chicago: University of Chicago Press.

[8] Shi, S. (1979). A Study on Chinese Four-Character Collocations. Chengdu: Sichuan People Press.

[9] Wang, T. H. (2007). The Formation and Development of Chinese Four-Character Collocations. Journal of Beijing Institute of Engineering, 1, 58-60

[10] Zhang, Y. (2006). Chinese Four-Character Collocations and their Translations. Shanghai Foreign Studies University.

[11] Zuo, Z. J. (2006). The Acquisition of Chinese Four-Character Collocations: Cognitive Perspective. China University of Oceanography.

Weidong Yang was born in Henan, China in 1972. He received his second bachelor from China School of Journalism, China in 1997.

He is currently a lecturer of English with the School of Foreign Languages, China University of Petroleum. His research interests include linguistics, and English language teaching.

Weiping Dai was born in Shenyang, China in 1956. He earned his M.A. degree in ELT from Essex University, UK in 1996.

$\mathrm{He}$ is currently a professor of English with the School of Foreign Languages, China University of Petroleum. His academic interests include linguistics, and English language teaching.

Lijia Gao was born in Jilin, China in 1960. She attained her M.A. degree in English Literature from Jilin University in 2004.

She is currently a professor of English with the School of Foreign Languages, Jilin University. Her academic interests include linguistics, and English language teaching. 